\title{
Henry Bauchau dans la critique italienne
}

État présent des études

\section{Michele Mastroianni}

\section{(2) OpenEdition}

Journals

\section{Édition électronique}

URL : https://journals.openedition.org/studifrancesi/2963

DOI : $10.4000 /$ studifrancesi.2963

ISSN : 2421-5856

Éditeur

Rosenberg \& Sellier

\section{Édition imprimée}

Date de publication : 1 juillet 2013

Pagination : 396-415

ISSN : 0039-2944

\section{Référence électronique}

Michele Mastroianni, « Henry Bauchau dans la critique italienne », Studi Francesi [En ligne], 170 (LVII I

II) | 2013, mis en ligne le 30 novembre 2015, consulté le 02 février 2023. URL : http://

journals.openedition.org/studifrancesi/2963; DOI : https://doi.org/10.4000/studifrancesi.2963

\section{(c) $($ ) $\odot \odot$}

Creative Commons - Attribution - Pas d'Utilisation Commerciale - Pas de Modification 4.0 International - CC BY-NC-ND 4.0

https://creativecommons.org/licenses/by-nc-nd/4.0/ 


\title{
Henry Bauchau dans la critique italienne. État présent des études
}

\begin{abstract}
2013, the year which marks the centenary of the birth of Henry Bauchau, serves as the opportunity for an investigation to discuss and compare, for the first time, studies carried out by Italian researchers on an author now considered by critics to be among the most important Francophone writers. Starting from elements of erudition within Italian literary criticism on Bauchau (Italian translations of his works, studies on his poetics, conference papers, reviews etc.), this study is meant to celebrate an influential author and to offer an overview of the reception of Bauchau's works in Italy.
\end{abstract}

Il 2013, anno in cui si celebra il centenario dalla nascita di Henry Bauchau, diventa qui motivo di un'indagine che, per la prima volta, discute e mette a confronto i dati relativi agli studi italiani, su quello che ormai la critica considera come uno degli autori di lingua francese fra i più importanti a livello internazionale. Partendo da elementi di erudizione (traduzioni italiane delle opere di Henry Bauchau, studi sulla sua poetica, atti di convegni, recensioni, ecc., all'interno del panorama critico d'Italia) si è voluto celebrare la figura di un autore di rilievo e offrire allo studioso una panoramica sulla ricezione, in territorio italiano, delle opere di questo scrittore.

La synthèse des données concernant la réception de l'œuvre d'Henry Bauchau en Italie, que nous proposons, a comme but principal de célébrer un auteur de premier plan sur la scène littéraire internationale, à cent ans de la date de sa naissance (22 janvier 1913), dans un moment où, surtout en Belgique et en France, les activités scientifiques autour de ses écrits abondent. Nous désirons, par là même, rendre hommage à cet écrivain, mort le 21 septembre 2012, et avec qui nous avons entretenu une correspondance amicale, par l'entremise de la revue «Studi Francesi», laquelle, en 2008 et en 2011, lui avait déjà accordé son attention.

1. S'il n'est pas question ici de souligner l'importance culturelle et littéraire d'Henry Bauchau en Belgique ${ }^{1}$, en France ${ }^{2}$ et dans plusieurs pays européens et non

(1) Nous rappelons, à ce propos, que les Archives et Musée de la Littérature de Bruxelles, dirigées par M. Quaghebeur, sont aujourd'hui un important pôle de recherche pour ceux qui étudient l'œuvre d'Henry Bauchau. Un centre auquel, depuis juin 2006, par volonté et concession de l'auteur, s'est joint un autre pôle d'investigation créé à l'Université catholique de Louvain (Louvain-la-Neuve). Il s'agit du Fonds Henry Bauchau, étant celui-ci aussi un centre de recherche de prééminence pour les études bauchaliennes. Ce dernier a été fondé par Myriam Watthee-Delmotte, qui en dirige les enquêtes scientifiques et organise les activités autour des œuvres de Bauchau. Les deux centres d'études conservent plusieurs documents manuscrits de l'auteur, dont beaucoup d'exemplaires, encore inédits, sont la preuve d'une richesse textuelle sur laquelle il est possible de mener des recherches intéressantes, soit dans la perspective philologique, soit dans la perspective de la critique génétique et de la réception/reconstitution d'un imaginaire poétique. Pour ce qui concerne les publications les plus importantes des deux centres mentionnés, parues ces dernières années, nous rappelons, au moins, AA. Vv., Les Constellations Impérieuses d'Henry Bauchau (Colloque de Cerisy-la-Salle, 21 31 juillet 2001), «Actes publiés sous la direction de M. Quaghebeur», Bruxelles, Archives et Musées de la Littérature («Collection Archives du futur»), 2003 et la publication des cinq premiers numéros (2008: L'atelier d'Henry Bauchau; 2009: Henry Bauchau et les arts; 2010: L'ancrage suisse; 2011-2012: 
européens ${ }^{3}$ qui, depuis quelques années, ont tout à fait reconnu la valeur de cet auteur $^{4}$ au niveau de la pensée 'humaniste' et philosophique dans l'époque charnière des $\mathrm{Xx}^{\mathrm{e}}$ et $\mathrm{XXI}^{\mathrm{e}}$ siècles, c'est, par contre, dans ce contexte-ci que la question de la présence et de l'importance poétiques de Bauchau en Italie mérite des observations pouvant aider à attirer l'attention de la critique sur sa production qui reste, malgré tout, encore assez négligée dans le panorama des études italiennes.

Si la réception initiale des écrits de cet écrivain, en Italie comme en Belgique et en France, passe surtout par la circulation de ses romans et non par ses recueils poétiques - il s'agit en effet d'un 'cas' éditorial à partir de Géologie $e^{5}$ - de son côté,

Henry Bauchau en traduction; 2013: Le temps du créateur) de la «Revue Internationale Henry Bauchau. L'écriture à l'écoute», sous la direction $\mathrm{M}$. Watthee-Delmotte et C. Mayaux (Louvain, Presses Universitaires de Louvain).

(2) En France, à Paris en particulier, c'est Catherine Mayaux qui a attiré l'attention sur cet auteur surtout par la coorganisation, avec Myriam Watthee-Delmotte, d'un congrès international: AA. Vv., Henry Bauchau poète, écrire pour habiter le monde, «Colloque international de l'Université Cergy-Pontoise (8-10 mars 2007)», Saint-Denis, Presses Universitaires de Vincennes, 2009.

(3) En témoignage de l'intérêt et du succès que les œuvres d'Henry Bauchau ont eu dans plusieurs pays du monde, nous signalons ici les traductions de ses livres, qui ont été publiées jusqu'à présent. Parmi les traductions allemandes: CEdipus unterwegs, par A. Neuschäfer, Biefeld, Cordula Haux Verlag, 1995; Attentives-Lauschende, par A. Neuschäfer, Aachen, Shaker Verlag, 2001 (il s'agit d'un florilège de poèmes); Instrumente der Freude, Wir wachten sebend auf, Der Baikal, Maas für Valentine, Der weiße Kieselstein, Das Werk, par R. Fischer, dans Encore une journée/Ein weiterer Tag, Rimbach, Éditions en forêt/Verlag im Wald, 2002 (il s'agit d'un florilège de poèmes). Pour les traductions en anglais: Oedipus on the road, par A.-M. Glasheen, New York, Arcade Publishing, 1994; pour les traductions en chinois: Edipusi piao liu ji, par H. Shuzhan, Pékin, Qunzhong, 1995. Parmi les traductions en espagnol: Edipo-Las puertas de Tebas, par L. López Morales, dans C. M. Pellicer - L. Lopez Morales (dir.), Literatura francófona: I. Europa, México, Fondo de Cultura económica, 1995; El árbol loco, par R. García López - B. Rey - Jouvin Manhes, dans AA. Vv., Bélgica hoy: Antología de la novela corta, Gran Canaria, Universidad de Las Palmas, 1997; El Desgarro, par R. García López, Gran Canaria, Universidad de Las Palmas, 2002; Antigona, par L. López Morales, México, Verdehalago, 2003; El niño azul, par T. F. Aúz - B. Eguibar, Valencia, Pre-Textos, 2008. Pour les traductions en galicien: Dependencia amorosa do poema, Dous poemas inéditos, Exercicio da mañá, Palabras do corpo adormecido, A xorba arella ou o soño de Freud, A sibila, O pensamento vexetal, par E. Araúxo, «Amastra-N-Gallar», 4 (outono,

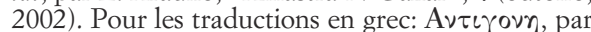

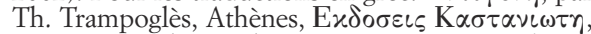
1998; parmi les traductions japonaises: Oidipusu no tabi, par Y. Miyahara, Tokyo, Shoshi-Yamada, 1994; Anchigone, par Y. Miyahara, Tokyo, ShoshiYamada, 2001. Parmi les traductions néorlandaises:
Gedichten uit: Het innerlijke China, par J. H. Mysjkin, dans Hanenveren van diverse pluimage. Levende franstalige poezie uit België, Gent, Poezie Centrum, 2004; Maalstroom, par K. Lauwerys, Amsterdam, Meulenhoff/Manteau, 2009; Het blawwe kind, par K. Lauwerys, Antwerpen, De Bezige Bij, 2011. Parmi les traductions portugaises: Edipo na estrada, par E. Grünewald, Rio de Janeiro, Lacerda Editores, 1998; Edipo en el camino, par H. H. García, Buenos Aires, Del estante Editorial, 2006; El niño azul, par H. H. García, Buenos Aires, Del estante Editorial, 2006. Parmi les traductions roumaines: Edip pe dru, par R. Lascu-Pop, Bucuresti, Editura Libra, 1997; Diotima sileii, par G. Marian, Bucureşti, Editura Libra, 2000. Parmi les traductions russes: Edip putnik, par O. Kysmoboù, Санкт-Петеръург, Инапресc, 1999; Antigóna, par O. Kysmoboù, Санкт-Петеръург, Инапресc, 2000. Pour les traductions en serbe: Periferijski bulevar, par A. Petrović, Belgrade, Arhipelag, 2010. Pour les traductions en tchèque: Oidipova Cesta, par V. Daňková, Praha, Česká expedice, 1994. Parmi les traductions turques: Oidipus Yollarda, par A. Bora, Istanbul, Metis Edebiyat, 1997; Antigone, par S. Dolanoğlu, Istanbul, Metis Edebiyat, 2002; Diotime ve aslanlar, S. Dolanoğlu, Istanbul, Metis Edebiyat, 2004; Mavi Cocuk, par S. Dolanoğlu, Istanbul, Metis Edebiyat, 2005 (pour les traductions signalées cf. la Liste des traductions en 2012, «Revue Internationale Henry Bauchau. L'écriture à l'écoute», 4 (2011-2012), pp. 51-55. Il s'agit d'un numéro monographique sur les problèmes de la traduction, concernant l'œuvre de l'écrivain (Henry Bauchau en traduction), et que Ch. Elefante a coordonné avec M. Watthee-Delmotte et C. Mayaux. On peut avoir aussi ces mêmes informations grâce à la consultation du site internet consacré à l'auteur: bauchau.fltr.ucl.ac.be, à la voix (Euvre/Traductions).

(4) Il serait suffisant, à ce propos, de signaler que le nom d'Henry Bauchau a été inclus, parmi les noms propres, dans l'édition de 2010 du dictionnaire Le Petit Robert (Paris, Editis). Du reste, les manuels de littérature française les plus récents ne manquent pas d'en signaler la présence parmi les auteurs qui font autorité entre les $\mathrm{XX}^{\mathrm{e}}$ et $\mathrm{XXI}^{\mathrm{e}}$ siècles.

(5) L'importance qu'Henry Bauchau accorde, depuis toujours, à la poésie - à partir de ses trentesix ans - est explicitée dans tous ses écrits théoriques, dans ses journaux et aussi dans la majorité des déclarations publiques abordant ce sujet. Or, si d'une part il s'agit d'une donnée indéniable, gravitant autour de son idéologie de l'écriture, au point qu'elle est au centre de l'élaboration poétique bau- 
l'attention italienne pour les œuvres de Bauchau nait grâce à l'intérêt de quelques chercheurs de l'Université de Bologne. En 1991', à Noci (Bari) est organisé un séminaire international, sous la direction de A. Soncini $\mathrm{Fratta}^{7}$, auquel participe Henry Bauchau. L'auteur apporte une contribution importante pour la lecture de ses œuvres, ainsi que pour la discussion sur les perspectives pionnières envisagées par les chercheurs.

La première réception sur les territoires belges et français des écrits de Bauchau - ainsi que nous l'avons souligné - a eu lieu par le biais des ouvrages romanesques. En Italie, les contributions du séminaire de Noci - il s'agit de la première attestation d'un intérêt scientifique pour l'œuvre de Bauchau - portent surtout sur des questions d'autobiographie et de réécritures mythiques, et bien marginalement sur des problématiques autour de la poésie de l'auteur. C'est Adriano Marchetti, spécialiste de littérature francophone, qui oriente, après 1991 et suite à la circulation des versions italiennes de quelques romans de Bauchau, publiées à partir de 1993, l'attention critique sur les poèmes et non sur les ouvrages en prose de notre écrivain. L'année 1999 - il s'agit de la date de publication de Dal naturale della mano ${ }^{8}$ (par

chalienne, il faut relever, d'autre part, que le grand succès arrive toujours par l'écriture en prose. Ce qui est vrai déjà à partir du premier recueil Géologie, dont la rédaction se conclut en 1957, même si la publication date de 1958 (Paris, Gallimard). En effet, bien que les attentes autour du succès de cette œuvre soient très fortes, et qu'avec Géologie Bauchau obtienne l'important prix littéraire Max Jacob (1959), il est indéniable que le succès du grand public n'arrive pas par ses poèmes. Dans ce cas, comme dans d'autres qui suivirent la date de parution du recueil Géologie, le succès espéré est déçu. Il faut attendre, au moins en Belgique et en France, l'année de la publication d'Edipe sur la route (Arles, Actes Sud, 1990), mais surtout d'Antigone (Arles, Actes Sud, 1997), c'est-à-dire l'extraordinaire succès éditorial et critique de ces romans, pour que Bauchau puisse se réjouir de reconnaissances envers ses poèmes. Le succès de Bauchau en poète arrive, au niveau international, en 2006 avec Nous ne sommes pas séparés. Poésie (Arles, Actes Sud) et en 2009 avec la publication de son recueil collectif Poésie complète (Arles, Actes Sud). À partir de ce moment, l'intérêt pour cet écrivain montant porte même vers l'appréciation et la réévaluation des poèmes publiés en précédence. Dans la perspective de la prééminence de la poésie sur la prose chez Bauchau, nous renvoyons à un passage qui synthétise de manière très claire son idéologie sur la nature de la poésie: «C'est dans le monde disloqué où l'angoisse m'avait fait entrer, dans ce labyrinthe de l'analyse qui n'avait pas de fil d'Ariane pour revenir en arrière que je me suis mis à écrire, ou peut-être à apprendre à écrire des vers [...]. Pourquoi des vers, pourquoi la poésie plutôt que la prose? Je me posais incessamment cette question. Pour y répondre il eût fallu savoir ce qu'était la poésie, ce que signifiait en moi les vers. Mais j'étais bien incapable de les penser, je pouvais seulement les vivre, et les éprouver comme une exigence intérieure. Il me semble aujourd'hui que je me suis tourné alors vers la poésie parce qu'elle vient de plus loin, d'une étendue plus profonde de l'histoire et de la préhistoire humaine. Parce qu'elle me reliait à des couches plus originelles de ma géologie personnelle» (cf. H. BAUCHAU, L'Écriture et la Circonstance, Louvain-la-Neuve, Presses Universitaires de Louvain UCL («Chaire de Poétique $2 \gg), 1988$, pp. 5-6). Encore un sentiment de déception accompagne la production théâtrale de Bauchau. S’il est vrai que les difficultés techniques sont à la base d'un choix précis de l'auteur, qui décide bientôt d'abandonner ce type d'écriture, il est vrai de même que le sentiment de faillite est également vécu de la part de Bauchau à propos de certaines de ses pièces. En effet, le 29 avril 2001, en parlant de Gengis Khan (Lausanne, Mermod, 1960) il dit: «Cette pièce a vraiment marqué mon entrée, non pas dans l'écriture, mais dans ma prise de conscience, encore bien incertaine, d'être un écrivain. C'est autour de cette œuvre que j'ai formé les plus grandes espérances et connu mes plus grandes déceptions» (cf. H. Bauchau, Passage de la BonneGraine, Arles, Actes Sud, 2002, p. 344).

(6) De toute manière, Bauchau capture l'intérêt du public international à partir des années 1990.

(7) AA. Vv., Henry Bauchau. Un écrivain, une ceuvre, «Atti del Centro di Studi sulla letteratura belga di lingua francese», (Terzo Seminario Internazionale, Noci 8-10 novembre 1991), a cura di A. SonCini Fratta, Bologna, Editrice CLUEB («Belœil», n 13$), 1993$.

(8) Ce volume présente en traduction italienne, précédée des textes français, un très riche florilège de quarante-huit poèmes de Bauchau, publiés dans Heureux les déliants. Poèmes (1950-1995), Bruxelles, Labor, «Espace Nord», 1995. Pour des raisons d'espace, nous devons renoncer à une description détaillée de cet ouvrage. De toute manière, nous renvoyons à l'étude de S. AmADORI, «Dal Naturale della mano» d'Adriano Marchetti ou la traduction comme 'épreuve' du dialogue avec l'inconscient créateur d'Henry Bauchau poète, «Revue Internationale Henry Bauchau. L'écriture à l'écoute», Henry Bauchau en traduction, 4 (2011-2012), pp. 78-90, qui analyse avec précision le travail traductif et 
A. Marchetti, Castel Maggiore, Book Editore) et aussi de L'innocenza dell'orecchio9 (par A. Marchetti, Bologna, Il Capitello del Sole) - marque la naissance d'une première historiographie sur la présence de Bauchau en Italie en tant que poète.

La divulgation successive des œuvres de cet auteur passera, d'une part, surtout à travers la traduction de quelques-uns de ses romans, de l'autre par des enquêtes sur sa production et sur l'idéologie élaborative étant à la base de sa poétique.

2. Si la première version italienne de l'œuvre d'Henry Bauchau concerne le roman Edipe sur la route ${ }^{10}$ (Arles, Actes Sud, $1990^{11}$; trad. it.: Edipo sulla strada, a cura di A. Marchetti ${ }^{12}$, Firenze, Giunti Editore, $1993^{13}$ ), il est indéniable le fait que la présence de cet écrivain en Italie commence à être significative à partir de la résonance internationale des romans CEdipe sur la route et Antigone ${ }^{14}$. C'est par la circulation de la traduction de ce dernier texte (Arles, Actes Sud, 1997; trad. it.: Antigone, a cura di A. Vitale, Firenze, Giunti Editore, 1999) que Bauchau éveille à la fois l'intérêt de la presse $e^{15}$ et élargit celui des chercheurs italiens ${ }^{16}$. Du reste, la version italienne d'Antigone en Italie, comme en Europe, réveille la curiosité du public spécialiste et du lecteur commun même à propos d'Edipe sur la route, publié - ainsi qu'on l'a signalé - en édition française en 1990 et en traduction italienne en 1993.

interprétatif d'A. Marchetti. Six ans auparavant, A. Marchetti avait publié, en traduction italienne, un florilège de poèmes de Bauchau (L'albero folle, «In forma di parole», 4 (ottobre-dicembre1993), pp. 143-148). En 1996 il fait sortir un deuxième florilège (Poesie, «In forma di parole», 2 (aprilegiugno 1996), pp. 196-215).

(9) Dans ce cas aussi, toujours pour des raisons d'espace, nous nous bornons simplement à relever que le but des fragments théoriques, autobiographiques ou de nature diaristique contenus dans cet ouvrage (Poète n'est pas maître chez lui; Dépendance amoureuse du poème; Fragments de Journal; Le Baikal), ainsi qu'A. Marchetti le souligne dans sa Postfazione (pp. 57-60), n'est pas celui de «tenter d'esquisser des profils d'une œuvre vaste, hétérogène et encore en cour d'élaboration. Simplement, on a voulu indiquer un trait du chemin, c'est-à-dire les quelques intonations de lecture que certaines réflexions, recueillies par l'auteur même pour nous dans son chantier de travail, nous suggèrent» (cf. A. MARChETTI, L'innnocenza dell'orecchio, cit., p. 57. La traduction est de nous).

(10) Le succès de cet ouvrage est attesté par le Prix triennal du roman et le Prix Antigone, décernés à Bauchau en 1990.

(11) Nous faisons toujours référence à la première édition des ouvrages de l'auteur.

(12) En 1993, A. MARChETTI est l'auteur d'une communication EEdipe sur la route d'Henry Bauchau. Le langage poétique et sa provenance, publiée dans C. VAN Rogger ANDReucci (dir.), Les nouveaux courants poétiques en France et en Grèce en 1970-1990, («Actes du Colloque International de Thessalonique, 21-24 septembre 1993», Pau, Presses Universitaires de Pau, 1994, pp. 249-256). En 1995 il fait sortir encore un travail sur Edipe dont le titre est Les rêves dans «CEdipe sur la route» d'Henry Bauchau, Rêve et poésie, «Recherches et Travaux», 47 (1995), pp. 203-
217. En 1998, après la publication de sa version italienne de 1993 d'CEdipe sur la route, Arles, Actes Sud, 1990 (trad. it.: Edipo sulla strada, Firenze, Giunti Editore), il publie un article sur La traduction comme exode. Notes en marge de la traduction d' «CEdipe sur la route», «Cahiers Henry Bauchau», 2 (octobre 1998), pp. 36-45. Quelques années plus tard, toujours sur la problématique des mythes chez Bauchau, il publie Le Labyrinthe: mythe de l'écriture, écriture du mythe, dans AA. Vv., Les Constellations Impérieuses d'Henry Bauchau, cit., pp. 288-306.

(13) Toujours en 1993, A. Marchetti publie un entretien avec Henry Bauchau (Peut-être que l'écriture va devenir plus bumaine que la parole: entretien avec Henry Bauchau, «Francofonia», 25 (1993), pp. 3-14), qui ouvre des perspectives intéressantes sur le tissu idéologique sous-jacent à l'écriture de l'auteur, et un article axé sur une problématique centrale chez Bauchau: celle de l'écoute (La fedeltà dell'ascolto, «In Forma di parole», 4 (ottobre-dicembre 1993), pp. 149-151).

(14) Pour ce roman, l'écrivain obtint deux prix prestigieux. Le premier en 1997 (Prix Rossel), le deuxième en 1999 (Prix des Lycéens).

(15) En particulier, la presse italienne commence à accorder de l'attention à cet auteur en 1994. En 2009, au moment où sort la traduction italienne de Le boulevard périphérique (Arles. Actes Sud, 2008), sous le titre de Il compagno di scalata (par Ch. Elefante, Roma, E/O, 2009), «La Repubblica» consacre, en l'espace d'à peine un mois, deux articles à Henry Bauchau. Le deuxième, celui du mois de mai, étant particulièrement intéressant, est axé sur des problématiques d'élaboration qui concernent le laboratoire poétique de Bauchau.

(16) En effet, entre 1999 et 2011 les traductions et les recherches sur l'œuvre d'Henry Bauchau prennent du relief parmi les études italiennes concernant la littérature francophone. 
Par ailleurs, toujours lié à l'élaboration d'une écriture mythique autour du cycle œdipien - une écriture par laquelle Bauchau essaie de «libérer les personnages de la prison du mythe, les transformant en personnages face auxquels se posent les problèmes de notre temps ${ }^{17}$ - est le recueil de récits Diotime et les lions (Arles, Actes Sud, 1991), dont la traduction, en Italie (Diotima e i leoni, a cura di G. Guglielmi, Firenze, Giunti Editore, 1993), sort parallèlement à celle d'Edipe sur la route. La parution en 1993 de la version de Diotime et les lions est suivie, un an après, d'une nouvelle édition italienne, florilège, celle-ci, de quelques récits de Bauchau publiés en textes autonomes avant 1994. L'édition italienne de ces récits, signalée dans La Repubblica par D. Pasti, le 26 octobre $1994^{18}$, porte le titre du premier texte qu'elle contient (Il ragazzo di Salamina, a cura di F. Trotta, Firenze, Giunti Editore, 1994). Il s'agit, dans l'ordre, de L'enfant de Salamine («La Revue générale», 3 (mars 1991), pp. 8192), L'arbre fou («La Revue générale», 2 (février 1993), pp. 91-96), Le Cri d'Antigone («La Revue générale», 8-9 (septembre 1993), pp. 23-28), Le temple rouge («La Revue générale», 11 (novembre 1994), pp. 7-16). La circulation de ces textes, surtout en Belgique et en France, est circonscrite dans la période qui va de 1991 à 1994. Par la suite, entre 1995 et 2001, l'attention de la critique italienne gravite encore autour de la traduction des œuvres de l'auteur. Ainsi, d'une part l'intérêt glisse sur son premier texte de théâtre, Gengis Khan (Lausanne, Mermod, 1960), de l'autre sur son premier roman, La Déchirure (Paris, Gallimard, 1960). Les années 1995 et 2001 sont liées à la publication des deux versions italiennes des textes mentionnés (Gengis Khan, a cura di G. Saverio Santangelo, Rimini, Panozzo Editore, 1995; La lacerazione, a cura di Ch. Elefante, Rimini, Panozzo Editore, 2001), même si dans ce canal privilégié d'investigation il reste un vide de cinq ans où aucun des écrits de Bauchau n'a fait l'objet de l'attention de la part des critiques italiens, sensibles pourtant aux problématiques de la traductions des ouvrages de l'auteur.

Cependant, peu de temps après la dernière version italienne du premier roman de Bauchau, sort en traduction un autre roman édité encore une fois par les soins de Ch. Elefante. Il s'agit de Le boulevard périphérique ${ }^{19}$ (Arles, Actes Sud, 2004; trad. it.: Il compagno di scalata, a cura di Ch. Elefante, Roma, Edizioni E/O, 2009), dont la version italienne est signalée dans «La Repubblica» par F. Frediani, le 25 avril 2009. Toujours en 2009, moins d'un mois après le compte-rendu de Frediani, «La Repubblica», dans la section Almanacco dei libri du 9 mai, fait sortir un article/interview, par F. Gambaro qui, d'une part rappelle aux lecteurs italiens le succès international et le contenu de Le boulevard périphérique, de l'autre insiste, en faisant appel au témoignage direct de Bauchau, sur le rôle joué par les figures mythiques de l'écrivain dans son système d'images, en liaison directe avec la valeur symbolique du rêve et à l'importance de la dimension onirique de ses œuvres par rapport à son travail de psychanalyste.

S'il est vrai que jusqu'à en 2009 les travaux de Ch. Elefante sont surtout axés sur des problématiques croisées - entre théorie de la traduction et objectivation formelle des principes idéologiques envisagés ${ }^{20}$ à partir de la traduction - il est vrai de même qu'en 2006, cette spécialiste de l'œuvre de Bauchau publie la traduction de

(17) Le passage cité est contenu dans «La Repubblica», sez. Almanacco dei libri, a cura di F. Gambaro (9 maggio 2009). La traduction est de nous.

(18) Par cette annonce, non seulement la presse italienne souligne l'importance littéraire de Bauchau, mais aussi le rôle de son expérience existentielle à l'intérieur de sa production. Ce qui correspond à la mise en valeur d'un élément reconnu aussi par la critique qui, depuis quelque temps, a vu dans cette compénétration entre vie et œuvre littéraire un point nodal du laboratoire poétique de Bauchau.

(19) Ce roman eut un grand succès auprès du public. C'est pour cette raison que Bauchau, la même année de la sortie de cette œuvre, obtint l'important Prix du Livre Inter 2008. 
quatre poèmes (Finestre di Presenza/Fenêtres de Présence. Finestre del Verde Aurorale/Fenêtres du Vert Levant. Mandala per una poesia/Mandala pour un poème. Figura del Tao/Signe du Tao, traduzione di Ch. Elefante, «Anterem. Rivista di Ricerca letteraria», 73 (dicembre 2006), pp. 52-61), contenus, à l'exception de Signe du Tao, dans le recueil Nous ne sommes pas séparés. Poésie (Arles, Actes Sud, 2006), montrant aussi un intérêt spécifique pour un genre littéraire dans lequel Bauchau s'essaie tout le long de sa vie. De 2006 à 2009, passant de la prose à la poésie, Ch. Elefante revient sur la prose de Bauchau, mais à travers une perspective différente qui se focalise sur des problématiques d'autobiographisme. Ce qui explique le choix de l'éditeur, portant sur la traduction de quelques pages importantes d'un texte diaristique Le Présent d'incertitude. Journal (2002-2005) (Arles, Actes Sud, 2007; trad. it.: La memoria e l'oblio, di Ch. Elefante, «Anterem. Rivista di Ricerca letteraria», 77 (dicembre 2008), pp. 90-111).

En guise de réflexion ultérieure sur les données bibliographiques qu'on vient de relever, nous offrons ci-dessous une grille récapitulative, qui permettra de faire, par la suite, des considérations sur la modulation des occurrences textuelles concernant la réception italienne des œuvres de l'auteur, à partir du Séminaire pionnier de Noci (1991), organisé par A. Soncini Fratta et publié (1993) dans la collection «Atti di ricerche, seminari e convegni», dirigée par R. Campagnoli.

AA. Vv., Henry Bauchau. Un écrivain, une oeuvre, «Terzo Seminario Internazionale, Noci, 8-10 novembre 1991», a cura di A. Soncini Fratta, Bologna, Editrice CLUEB, 1993

$\begin{array}{lll}\text { TEXTE FRANÇAIS } & \text { ANNÉE DE } & \text { VERSION } \\ & \text { PUBLICATION } & \text { ITALIENNE } \\ \text { Gengis Khan } & \mathbf{1 9 6 0} \text { (Lausanne, Mer- } & \text { Gengis Khan } \\ & \text { mod) } & \\ \text { La Déchirure } & \mathbf{1 9 6 6} \text { (Paris, Gallimard, } & \text { La lacerazione } \\ & 1966 \text { ) } & \\ \text { Edipe sur la route } & \mathbf{1 9 9 0} \text { (Arles, Actes Sud) } & \text { Edipo sulla strada }\end{array}$

ANNÉE DE
PUBLICATION

1995 (Rimini, Panozzo Editore $)^{21}$

2001 (Rimini, Panozzo Editore $)^{22}$

1993 (Firenze, Giunti Editore $)^{23}$
(20) Bien évidemment - toujours à propos des textes de Bauchau que Ch. Elefante a traduits - les principes théoriques suivis, notamment pour $\mathrm{La}$ Déchirure, mais probablement aussi ceux qui ont été utilisés pour les autres traductions, sont argumentés dans Ch. Elefante, Tradurre «La Déchirure» ecc., cit.

(21) Il est très intéressant de savoir combien d'exemplaires ont été vendus et ont circulé en Italie, afin d'avoir une idée de la fortune des œuvres de Bauchau dans notre territoire. Pour cette raison, nous avons contacté l'Éditeur Panozzo, lui demandant les chiffres des volumes ici concernés. À ce sujet, je tiens à remercier M. Massimo Panozzo qui a accepté de nous fournir les informations demandées. Le numéro d'exemplaires vendus de Gengis Khan correspond à un total de 377.

(22) Le nombre d'exemplaires vendus de La lacerazione s'élève à 21 .

(23) Pour les mêmes raisons énoncées supra (note 21), nous avons contacté l'Éditeur Giunti, lui demandant les données chiffrées se rapportant aux volumes ici concernés. Je remercie Mme Elena Orsini qui a bien voulu nous aider dans cette recherche. C'est à elle qu'on doit les données précises, que nous soumettons à la réflexion du lecteur. Le numéro d'exemplaires vendus d'Edipo sulla strada est le suivant: 1993 (3171 exemplaires); 1994 (113 exemplaires); 1995 (138 exemplaires); 1996 (0 exemplaires); 1997 (92 exemplaires); 1998 (114 exemplaires); 1999 (107 exemplaires); 2000 (133 exemplaires); 2001 (71 exemplaires); 2002 (29 exemplaires). Le total d'exemplaires vendus s'élève à 3968. Bien que les informations données par l'Éditeur s'arrêtent, dans tous les cas, à l'année 2002, les chiffres dont on dispose, permettent de faire quelques observations générales et, surtout en considération des données que nous fournissons sur les autres ouvrages de Bauchau traduits en italien, de relever que ce roman - parmi ceux qui ont été publiés chez Giunti - est celui qui a eu le plus de succès. Ce qui confirme la fortune européenne 


\section{Diotime et les lions}

L'enfant de Salamine;

L'arbre fou; Le cri

d'Antigone; Le temple

rouge

\section{Antigone}

[nous ne remettons pas ici à l'attention du lecteur les quarante-huit titres des textes concernés par des raisons d'espace, mais aussi parce qu'il s'agit de fragments tirés d'éditions d'ouvrages différents]

Poète n'est pas maître chez lui; Dépendance amoureuse du poème; Fragments de Journal; Le Baikal

Fenêtres de Présence; Fenêtres du Vert Levant; Mandala pour un poème; Signe du Tao ${ }^{27}$

Le Présent d'incertitude

Le boulevard périphérique
1991 (Arles, Actes Sud) Diotima e i leoni

1991 («La Revue générale», $\mathrm{n}^{\circ} 3$, mars, pp. 81-92); 1993 ( $\ll \mathrm{La}$ Revue générale», $\mathrm{n}^{\circ} 2$, février, pp. 91-96); 1993 («La Revue générale», $\mathrm{nn}^{\circ}$ 8-9, septembre, pp. 23-28); 1994 ( $\mathrm{La}$ Revue générale», $\mathrm{n}^{\circ} 11$, novembre, pp. 7-16)

1997 (Arles, Actes Sud)

[nous ne remettons pas ici à l'attention du lecteur les données des textes concernés, parce qu'il s'agit de fragments textuels tirés d'éditions et d'ouvrages différents]

2006 (Nous ne sommes pas séparés. Poésie, Arles, Actes Sud)

2007 (Arles, Actes Sud)

2008 (Arles, Actes Sud)

\section{Il ragazzo di Salamina}

Antigone

Dal naturale della mano [florilège de quarantehuit poèmes]
1993 (Firenze, Giunti Editore $)^{24}$

1994 (Firenze, Giunti Editore $)^{25}$
L'innocenza dell'orecchio [fragments théoriques, autobiographiques ou de nature diaristique]

Finestre di Presenza; Finestre del Verde Aurorale; Mandala per una poesia; Figura del Tao

La memoria e l'oblio [il s'agit d'un florilège de quelques passages, tirés $\mathrm{du}$ journal intime en question]

Il compagno di scalata
1999 (Firenze, Giunti Editore $)^{26}$

1999 (Castel Maggiore, Book Editore)
1999 (Bologna, Il Capitello del Sole)
2006 («Anterem. Rivista di Ricerca letteraria», $\mathrm{n}^{\circ} 73$, dicembre, pp. 52-61)

2008 («Anterem. Rivista di Ricerca letteraria», $\mathrm{n}^{\circ} 77$, dicembre, pp. 90-111)

2009 (Roma, Edizioni $\mathrm{E} / \mathrm{O})^{28}$
qu'Edipe sur la route a eu dans le monde.

(24) Le numéro d'exemplaires vendus de Diotima e $i$ leoni est le suivant: 1993 (1296 exemplaires); 1994 (1088 exemplaires); 1995 (147 exemplaires); 1996 (0 exemplaires); 1997 (51 exemplaires); 1998 (112 exemplaires); 1999 (102 exemplaires); 2000 (0 exemplaires); 2001 (0 exemplaires); 2002 (0 exemplaires). Le total d'exemplaires vendus s'élève à 2796.

(25) Malheureusement, à cause de l'absence d'un fichier papier de 1994, permettant de repérer les informations sur les ventes de Il ragazzo di Salamina, l'Éditeur Giunti, dans la personne de Mme Elena Orsini, n'a pas pu nous soumettre les données demandées.

(26) Le numéro d'exemplaires vendus d'Antigone est le suivant: 1999 (1891 exemplaires); 2000 (201 exemplaires); 2001 (0 exemplaires); 2002 (0 exemplaires). Le total d'exemplaires vendus s'élève à 2092.

(27) Ce dernier poème, en réalité, n'est pas contenu dans le recueil Nous ne sommes pas séparés. Poésie. Toutefois, dans l'édition collective de poèmes (H. Bauchau, Poésie complète, Arles, Actes Sud, 2009, p. 34), que Bauchau publie trois ans plus tard par rapport à la sortie de ce recueil, est présent un texte qui porte comme titre Tao. Il pourrait être que la traductrice disposait à ce moment donné d'un texte inédit dont le titre, dans sa version définitive, a été changé par la suite. Ce qui pourrait expliquer cette dissonance d'intitulations.

(28) Toujours pour les mêmes raisons, nous avons contacté l'Editeur E/O. Mme Maddalena Gentili, à qui vont nos remerciements, nous a communiqué que le nombre d'exemplaires vendus jusqu'à présent de Il compagno di scalata s'élève à 
Pour un cadre général plus précis, nous signalons également, dans le schémas suivant, les comptes-rendus des ouvrages de l'auteur, publiés dans la presse italienne:

\begin{tabular}{|c|c|c|c|}
\hline $\begin{array}{l}\text { TITRE DU } \\
\text { COMPTE-RENDU }\end{array}$ & AUTEUR & NOM DU QUOTIDIEN & $\begin{array}{l}\text { ANNÉE DE } \\
\text { PUBLICATION }\end{array}$ \\
\hline In viaggio con Edipo & D. Pasti & $\begin{array}{l}\text { La Repubblica. } \\
\text { Sezione cultura }\end{array}$ & 1994 (26 ottobre) \\
\hline Un caso di memoria & F. Frediani & $\begin{array}{l}\text { La Repubblica. } \\
\text { Sezione cultura }\end{array}$ & 2009 (25 aprile) \\
\hline Henry Bauchau & F. Gambaro & $\begin{array}{l}\text { La Repubblica. } \\
\text { Almanacco dei libri }\end{array}$ & 2009 (9 maggio) \\
\hline $\begin{array}{l}\text { Henry Bauchau, una } \\
\text { vita di lotte }\end{array}$ & A. Tito & L'Unità. Sezione speciali & 2012 (22 settembre) \\
\hline
\end{tabular}

Ainsi que le bilan final le montre, non seulement la réception massive des écrits de Bauchau passe, en Italie, par la publication de romans en traduction - on vient d'insister sur cet aspect -, mais aussi par un intérêt scientifique qui se construit à partir d'Edipo sulla strada (1993), et qui continue jusqu'à en 2009, avec la publication de Il compagno di scalata. Sur les neuf versions italiennes, quatre concernent des romans, deux des récits, tandis que les trois qui restent se repartissent ainsi: une traduction d'une pièce théâtrale, quatre versions italiennes de quatre poèmes tirés $\mathrm{du}$ recueil Nous ne sommes pas séparés. Poésie $e^{29}$ et la traduction en guise d'exemplum de quelques pages diaristiques du journal Le Présent d'incertitude ${ }^{30}$. En outre, si une régularité significative d'intérêt de la part de la critique italienne peut être retenue entre les années 1993-1995 (cinq sont les ouvrages de Bauchau qui, dans cette période, sortent en traduction), il est vrai de même que cette constante critique et éditoriale s'interrompt par la suite. En effet, l'on assiste à une période d'absence de trois ans, entre 1996 et 1998, qui nous fait glisser vers 1999, année marquée par le succès international du roman Antigone et, dans notre cas, par la circulation de la version italienne de cet ouvrage. Par la suite, si l'année 2000 ne laisse enregistrer aucune publication de l'auteur en Italie, un nouvel manque est relevé entre 2002 et 2005. Plus tard, par contre, en 2006 - ainsi qu'on peut le remarquer par le premier schémas ci-dessus - Bauchau est présent sur le territoire italien à travers la traduction de quatre poèmes édités en France au cours de la même année. À l'absence de 2007 fait suite une présence en 2008, par la traduction d'extraits significatifs (Le Présent d'incertitude) et par la dernière publication datée de 2009 (Il compagno di scalata), qui conclut ses passages littéraires en traduction italienne ${ }^{31}$.

Ce qui nous semble aussi digne d'être signalé - à côté de ces quelques remarques autour des périodes où il est possible de relever une présence plus significative de Bauchau en Italie, parallèlement, bien sûr, aux moments où il est absent de la scène critique et littéraire - c'est, nous semble-il, la donnée concernant l'écart temporel entre la sortie de son premiér écrit en Suisse (Gengis Khan, 1960) et la publication de la première version italienne (1993) d'un ouvrage ne correspondant pas, du reste, à la sortie de la pièce de théâtre mentionnée. En effet, si par Gengis Khan Bauchau fait sa première apparition dans le monde littéraire suisse et, par la suite, dans celui de la Belgique et de la France, c'est par contre par la traduction d'Edipe sur la route (trad. it.: Edipo sulla strada, Giunti Editore, 1993) que commence en Italie la fortune de l'auteur ${ }^{32}$.

1756, achetés surtout dans la région milanaise.

(29) Pour les références précises voir les données contenues dans la première grille.

(30) Cf. la note précédente.
(31) Cf. la note précédente.

(32) Il suffit de réfléchir sur les données concernant les ventes, en Italie, de cet ouvrage. Cf. supra, la note 23 . 
3. La fortune de Bauchau en Italie pourtant suit un double parcours. D'une part, la voie de la traduction de ses œuvres est un canal privilégié, permettant à l'auteur de rejoindre le public non spécialiste. D'autre part, ses écrits - qui se construisent toujours sur un substrat autobiographique, dont émerge une idéologie précise d'écriture, faisant appel à des formes de pensée différentes qui impliquent la superposition de discours herméneutiques hétérogènes, sur la base d'un mouvement exégétique complexe (de la vie à la psychanalyse, de la phénoménologie du rêve à un imaginaire puisant aussi dans l'existentiel, de la littérature à l'art, de la philosophie à la théorie de l'écriture, de la spiritualité catholique à une spiritualité 'universelle' etc.) - attirent l'attention des critiques, dès 1991.

À Noci - la référence est celle du séminaire dont nous avons parlé33 - se retrouvent des spécialistes de littérature française contemporaine, pour faire en sorte que les journées d'études soient une occasion de découverte et de débat, autour de l'œuvre de Bauchau, qui participe aux discussions. En effet, la participation de Bauchau aux travaux a beaucoup apporté aux enquêtes envisagées et aux résultats scientifiques obtenus, non seulement parce qu'il «a dénoué devant son public, la signification de situations et de moments dont il n'avait jamais parlé avant», mais aussi parce que les trois journées de ce séminaire lui ont permis de «parler de ses positions politiques, religieuses et sociales, en rapport avec l'époque de la seconde guerre mondiale» ${ }^{34}$. Bien évidemment, la présence et l'intervention de l'auteur au fil des communications et des réflexions finales se croisent avec les perspectives des recherches présentées, axées sur des données d'autobiographisme, d'imaginaire, de réception historique, contextuellement à la vie et à l'œuvre de l'écrivain, s'entremêlant avec le discernement sur la réélaboration des mythes anciens ou de personnages mythiques. Il se pourrait que - dans la perspective historique évoquée, une perspective ayant des rapports très forts et importants avec la dimension littéraire concernant la vie de l'auteur - sans rien enlever à la valeur cohérente générale de l'ensemble des communications, l'intervention de l'historienne Anne Morelli, à cette époque ${ }^{35}$, soit celle qui met en cause davantage Bauchau et, d'une certaine manière, la réception critique de ses écrits. En outre, l'intervention de Morelli - nous semble-t-il - agit comme une sorte de clé de lecture par rapport à la sémantique textuelle et à la symbolique gravitant autour des œuvres de Bauchau. Ce qui est encore plus vrai, si l'on considère qu'en 1991 les études sur notre écrivain, dans la perspective historique, étaient encore absentes. En effet, il faut attendre, en particulier, les années 2002 et 2008 pour que la critique puisse appuyer ses recherches et encadrer l'auteur sur la base de données historiques précises, fondamentales pour une nouvelle contextualisation de la poétique de Bauchau et des rapports qu'entretiennent ses écrits avec ses expériences de vie.

C'est dans M. Watthee Delmotte (dir.), Bauchau avant Bauchau. En amont de l'œuvre littéraire, Louvain-la-Neuve, Bruylant-Académia, 2002, et dans G. DUchenne - Vincent Dujardjn - Myriam Watthee-Delmotte, Henry Bauchau dans la

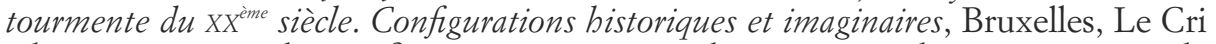
édition, 2008, que les configurations poétiques, historiques et littéraires autour de l'auteur se croisent, pour prendre de nouvelles directions. En tout cas, ainsi que nous l'avons souligné, l'étude de Morelli, communiquée en 1991 et publiée dans les Actes

(33) Pour les références et pour d'ultérieurs éclaircissement cf. supra, $\ 1$ et la note 7.

(34) Cf. R. Campagnoli, Presentazione, in Aa. Vv., Henry Bauchau. Un écrivain, une ouvre, cit., pp. 7-8, ici p. 7. C'est nous qui traduisons.

(35) Il nous semble très important, à l'intérieur de cette tentative de reconstruction de la présence de Bauchau en Italie dans une perspective critique, de mettre en relief le fait que A. Morelli, peut-être la première en Europe, a abordé la question de la participation active de Bauchau à la vie politique de la Wallonie entre les deux guerres et, notamment, à l'époque de la deuxième guerre. 
de 1993, ouvrait alors des perspectives de recherches inédites ${ }^{36}$, surtout à la lumière des réponses que Bauchau voulut donner aux remarques de Morelli.

Évidemment, dans ce contexte, nous ne pouvons pas offrir un examen détaillé de toutes les interventions des journées de Noci - ce qui, du reste n'est pas non plus le but de cette étude -, mais, par contre, nous pouvons ici, en guise d'information générale, signaler les repères bibliographiques des travaux contenus dans ce volume. Ces mêmes contributions qui, tout en fonctionnant en tant qu'instrument bibliographique rétrospectif, se sont imposées et s'imposent comme des données importantes par rapport aux recherches suivantes, celles menées jusqu'à présent: R. CAMPAGNOLI, Presentazione al terzo numero di «Beløil», pp. 7-8; M. VAN DE KerCKHOVE, Avant propos, pp. 11-14; J. TORDEUR, Le caractère épique dans le rapport de l'écrivain avec lui-même, pp. 15-22; A. MORELLI, La guerre dans l'œuvre et la vie d'Henry Bauchau, pp. 23-38; H. Bauchau, Note sur la communication de M.me Anne Morelli, pp. 3942 ${ }^{37}$; G. HenRot, «L'Escalier bleu» d'Henry Bauchau: le pari du rythme, pp. 45-80; A. GonzÀlez-Salvador, Tout dire du dedans. À propos d'Henry Bauchau, de la vérité et de la fiction, pp. 81-91; G. Saverio Santangelo, «Gengis Khan»: Dal Mito alla Storia, fra autobiografia e drammaturgia, pp. 93-126; N. MikLos, «Gengis Khan» ou le rêve d'être homme, pp. 127-143; S. FERRARI, «Gengis Khan»: protohistoire d'une mutation sociale, pp. 145-159; J. PAQUE, La question du genre ou les surprises $d u$ roman, pp. 161-175; P. HALEN, «La Déchirure», roman de la décolonisation? Notes sur l'œuvre d'Henry Bauchau et son inscription dans l'bistoire, pp. 177-200; J. GousSEAU, Pouvoir et société dans le «Régiment Noir», pp. 201-221; B. TshibOLA KalenGaY - K. MunzenZA Willy - K. ThiO, Le rôle et l'image du Noir dans «Le Régiment Noir» d'Henry Bauchau, pp. 223-240; M. Watthee-Delmotte, De l'aveuglement au sens, Edipe et l'écrivain sur la route, pp. 241-254; A. MARCHETTI, Il silenzio di Edipo e l'inviolabilità dell'appello, pp. 255-268; J. ROBAEY, Fonction et fonctionnement du mythe classique. De Sophocle à Bauchau, pp. 269-288; R. JouAnNy, Les lacunes du récit mythique dans «CEdipe sur la route»; CH. ELEFANTE, La responsabilité d' Edipe, du multiple à l'un, pp. 313-333; M. AmatUlli, Du Clairchantant au Clairvoyant: (auto) genèse d'une création, pp. 335-355; A. SONCINI-FraTTA, L'Edipe 'bachofien' de Bauchau, pp. 357-366; H. Bauchau, Notre Italie, pp. 367-369; A. M. ZavatTi, Bibliografia, pp. 371-382.

Or, deux ans après la parution des Actes du séminaire de Noci, en 1995 , A. Soncini Fratta réunit, dans le volume Jeux de langue, jeux d'écriture (collection «Atti di ricerche, seminari e convegni», Bologna, Editrice CLUEB, «Belœil», n 16) les études de quelques spécialistes de francophonie, pour «rendre hommage au mythe de la spécificité 'linguistique' de la littérature belge de langue française. C'est de mythe qu'il est question dans la mesure où cette spécificité n'est pas fondamentale, si l'on considère les tendances à la déformation linguistique présentes dans toutes les littératures européennes. [...] S'il a été possible de poser l'hypothèse d'une 'belgité, en tant que spécificité beaucoup plus vaste de la 'belgité' purement linguistique, cela s'est fait par le truchement du mythe de la spécificité linguistique» ${ }^{38}$.

Dans ce cadre argumentatif s'insère un article de Ch. Elefante (La scrittura del sogno della scrittura. A proposito di Henry Bauchau, pp. 129-151), où la cher-

(36) Une deuxième communication est axée sur des recherches portant aussi sur quelques aspects historiques. Il s'agit, en particulier, du travail de P. HALEN, «La Déchirure», roman de la décolonisation? Notes sur l'œuvre d'Henry Bauchau et son inscription dans l'bistoire, pp. 177-200.
(37) À la suite de l'intervention d'Henry Bauchau, il y a une réponse qui porte le titre de Anne Morelli ajoute. Il s'agit d'une réplique de Morelli aux observations que Bauchau relève, à partir de l'étude de cette historienne (cf. AA. Vv., Henry Bauchau. Un écrivain, une auvre, cit., p. 43).

(38) Cf. R. Campagnoli, Présentation, in ibid., 
cheur - passant des questions mythiques de 1995, gravitant autour d'CEdipe ${ }^{39}$, mais aussi de ses essais en tant que traducteur de l'œuvre de Bauchau ${ }^{40}-$ met en évidence les rapports existant entre Jour après jour. Journal (1983-1989) et la genèse d'Edipe sur la route. Il s'agit d'une genèse liée au monde onirique de Bauchau, à son expérience comme psychanalysé et psychanalysant, et aux renvois incontournables à la réalité du rêve, centrale, celle-ci, aussi bien dans l'idéologie poétique de l'écrivain que dans son esthétique. En 2000, par contre, après avoir dressé des enquêtes ultérieures sur le personnage d'Edipe, dans De l'Edipe travesti à la renaissance du Père en Amérique, la patrie mytique des fils, «Cahiers Henry Bauchau», 3 (juin 2000), pp. 12-20, elle revient sur des problématiques de théorie de la traduction, par une perspective de recherche linguistique axée sur la rhétorique et la stylistique de l'écriture de Bauchau. Cette recherche, pourtant, ne néglige pas de s'interroger sur le croisement exégétique entre nœuds littéraires et problématiques formelles de l'imaginaire et du langage de l'auteur ${ }^{42}$. C'est ainsi que Ch. Elefante publie Tradurre «La Déchirure» di Henry Bauchau. Metodo e prassi (Torino, L'Harmattan Italia, «Indagini e Prospettive», $2000)^{43}$. Quelques années plus tard, la chercheur, élargissant son axe d'étude autour de la présence mythique chez Bauchau, soutient une thèse de doctorat (Mito e luoghi dell'immaginario di Henry Bauchau, Bologna, 2006) qui à présent est, malheureusement, encore inédite. En 2009 elle est l'auteur de La voix du corps, la voix féminine dans l'ceuvre narrative d'Henry Bauchau, dans C. Mayaux - M. Watthee-Delmotte (dir.), Henry Bauchau, écrire pour habiter le monde, Vincennes, Presses Universitaires de Vincennes («L'Imaginaire du texte»), 2009, pp. 147-160, tandis qu'en 2012, reprenant les enjeux du discours entamé par la publication du texte mentionné (Tradurre «La Déchirure» etc.), toujours au sujet de la traduction en tant que lieu de réflexion théorique et moment d'essai sur le texte littéraire, Ch. Elefante partage avec M. Watthee-Delmotte et C. Mayaux la responsabilité d'un projet de recherche, dont les résultats ont été recueillis et publiés dans la «Revue Internationale Henry Bauchau. L'écriture à l'écoute», 4 (2011-2012), pp. 5-20544. C'est dans ce même numéro que

p. 8 .

(39) Сh. Elefante, La responsabilité d'Edipe, du multiple à l'un, dans AA. Vv., Henry Bauchau. Un écrivain, une ouvre, cit., pp. 313-333.

(40) Pour les références précises voir les données contenues dans la première grille, au $₫ 2$.

(41) Bruxelles, Les Éperonniers, 1992.

(42) En 2003 Ch. Elefante publie «Amour Diotime»: une lecture intertextuelle, dans Les Constellations Impérieuses d'Henry Bauchau, cit., pp. 212-237. En 2004 elle est aussi l'auteur de deux travaux très intéressants (Henry Bauchau e la scrittura, tra realtà, inconscio e vita onirica, «Tratti», 67 (autunno 2004), pp. 6-17; Henry Bauchau et Jean El Moubouv Amrouche: bistoire d'une amitié poétique, dans AA. Vv., Henry Bauchau, une poétique de l'espérance «Actes du Colloque International de Metz publiés sous la direction de P. Halen - R. Michel - M. Michel (6-8 novembre 2002)», Berne, Peter Lang, ( ${ }^{\circ} 7$ «Recherches en littérature et spiritualité»), 2004, pp. 41-62) qui annoncent, d'une certaine manière, la perspective littéraire qui s'ouvre avec ses recherches sur l'imaginaire chez Bauchau.

(43) L'introduction de cette étude (pp. 6-14) et le premier chapitre (La Déchirure en abîme, pp. 1555) portent sur des questions de nature littéraire, tandis que le deuxième chapitre (Tradurre la lacera- zione, pp. 56-118) est construit sur un discours par lequel l'auteur discute des problématiques de traduction textuelle, sur la base d'exemples concrets. Pour des informations détaillées sur ce travail, voir le compte-rendu de L. RegGiani, «La Déchirure» di Henry Bauchau ecc., cit., Henry Bauchau. Voix et vocation de l'écriture, «Francofonia. Studi e ricerche sulle letterature di lingua francese», 42 (primavera 2002), pp. 152-156.

(44) Ce numéro se structure de la manière suivante: une première section (Inédits. Dossiers de traduction, pp. 11-22) est consacrée à la traduction du poème de Bauchau, Tentatives de louange, en plusieurs langues (deux traductions anglaises; deux traductions allemandes; une traduction néerlandaise; une traduction italienne; une traduction espagnole; une traduction roumaine; une traduction ukrainienne; une traduction russe). Une deuxième section (Poèmes inédits d'Henry Bauchau, pp. 2325) offre, pour la première fois, trois poèmes de l'auteur: L'Enfant rieur, Exercice du soir et $A u$ Déliant. La troisième section (Dans la compagnie des troubadours: extraits de la correspondance inédite entre Henry Bauchau et Emilio Araúxo, pp. 2646) porte sur des pages de correspondance diaristique entre les deux écrivains (E. Araúxo, natif de l'Orens, l'une des provinces de la Galice, est aussi ethnographe et professeur de philosophie) 
Ch. Elefante (La voix du traducteur et l'écho de la traduction, pp. 56-67), prend en charge un travail d'enquêtes sur la traduction, à travers un double axe de recherche, bâti encore une fois sur des problématiques de genèse littéraire en perspective critique $^{45}$. Un travail portant sur des questions de narration d'un côté et de stylistique de l'autre, qui relève des échos entre La Déchirure et Le boulevard périphérique (Arles, Actes Sud, 2008), tout en étudiant ces passages qui fonctionnent, vis-à-vis des deux romans, en matériel d'intertextualité poétique et linguistique.

Encore par les soins de l'École bolonaise, représentée une fois de plus de l'expérience d'A. Marchetti en matière bauchalienne, en 2002 sort un numéro monographique: Henry Bauchau. Voix et vocation de l'écriture, «Francofonia. Studi e ricerche sulle letterature di lingua francese», 42 (primavera 2002), pp. 1-13646. Ainsi qu'A. Marchetti le souligne, la perspective d'enquête autour de laquelle s'organise ce volume part de la constatation que chez Bauchau «on peut repérer deux tendances parallèles: la fiction et le poème. Que le lien les unit, là est la question. Certes, l'écrivain manifeste un goût marqué pour certaines périodes, pour certaines cultures: l'Amérique de la Guerre de Succession, l'Antiquité grecque, l'Asie de Lao-Tseu, la Chine médiévale et celle de Mao Tsé-toung, aussi bien qu'une Chine intérieure. Une réalité imaginaire, en partie involontaire dans le rêve, qui s'impose à l'écrivain comme une cartographie visionnaire et désirante», mais aussi du souhait que ce même numéro monographique puisse «être tout à la fois une contribution au développement de la recherche et une invitation à méditer le mystère qui accompagne la création littéraire, sans prétendre toutefois baliser l'étendue de cette œuvre [scil. d'Henry Bauchau] considérable» ${ }^{47}$.

Les contributions, dont plusieurs abordent la problématique concernant l'inspiration poétique et la rédaction des poèmes, gravitent aussi autour de deux textes d'Henry Bauchau et font suite au schéma herméneutique et scientifique suivant: A. Marchetti, Envoi, pp. 5-7; N. Huston, Bouquet Bauchau, pp. 9-13; M.-Cl. Boons, Henry Bauchau le rêveur, le poète, pp. 15-25; G. MARIAN, Mystique du corps: cinq poèmes d'Henry Bauchau, pp. 27-36; G. Henrot, «Mélopée viking»: Récit, prophé-

qui s'écrivent, puisque - ainsi que C. Mayaux le souligne - Araúxo «s'adresse à Henry Bauchau en 2000 pour le solliciter dans le cadre d'un volume qu'il prépare aux Edicions do Cumio présentant soixante-dix-neuf poètes du monde entier et des entretiens menés avec eux, comme celui qu'il propose à distance à Bauchau. [...] Au-delà des échanges de poète à poète et des questions de lecture et d'interprétation qui surgissent çà et là, cette correspondance montre certains aspects de la vie culturelle en Galice autour de la poésie et de l'édition de poésie, dont Emilio Araúxo est un auteur éminent par ses travaux et ses fonctions d'éditeur» (cf. le numéro 4 de la «Revue Internationale Henry Bauchau. L'écriture à l'écoute», cit., p. 26). La section qui suit (Dossier thématique. Henry Bauchau en traduction, pp. 47-146), celle organisée par Ch. Elefante, est axée sur des problématiques qui relèvent tout particulièrement de l'intérêt pour la traduction littéraire, puisque «les traductions restent un objet d'études largement inexploré et insuffisamment approfondi. Ce numéro de la Revue pose au centre de l'attention des spécialistes l'œuvre d'Henry Bauchau en traduction, en suggérant, à travers des études de traducteurs et de chercheurs, des réflexions qui engagent plusieurs pistes herméneutiques liées à l'analyse critique des traductions existantes ou à l'expérience de la traduction elle-même» (cf. ibid., p. 48). La quatrième section (Varia, pp. 147-192) gravite autour de quatre études qui ne concernent pas vraiment la problématique envisagée, mais qui sont axées sur des questions très intéressantes de la poétique de Bauchau (J. LAMBERT, Le récit mythique de «Géologie»: l'émergence d'une parole-trace, pp. 149-159; M. DuBoIs, Puissance auto-affective et pathétique de l'écriture poétique, pp. 160-171; I. VAnQueTEM, Sujet individuel, sujet collectif? La libération dans l'œuvre d'Henry Bauchau, pp. 172181; M.-C. TOMAsI, Quand la vague rencontre le roc: l'émergence de l'île bauchalienne, pp. 182-192). La dernière section (Actualités, pp. 194-202) contient les références des travaux critiques sur Bauchau, parus entre 2010 et 2011, ainsi que les références concernant les derniers écrits de l'auteur et celles qui informent, par contre, sur les expositions organisées autour de son œuvre.

(45) Sur cet aspect, voir les notices données dans le $\$ 3$, concernant l'article de CH. Elefante, La scrittura del sogno ecc., cit.

(46) Une partie considérable de la section conclusive de ce numéro monographique est consacrée aux comptes-rendus des œuvres de Bauchau (cf. Henry Bauchau. Voix et vocation etc., cit., pp. 137-158). 
tie, allégorie, pp. 37-58; M. WatTheE-Delmotte, En filigrane de l'ouvre d'Henry Bauchau: le motif du Graal, pp. 59-75; A. NeusCHÄFER, Tra le reti della lingua: note sulla poesia di Henry Bauchau, pp. 77-85; A. SonCINI FRATTA, Le rapport au père: un par(ad)i(s) impossible à propos du «Prométhée enchaîné» d'Henry Bauchau, pp. 87-98; I. GABOLDE, La lumière de «L'Enfant bleu», une écoute entre prose et poésie, pp. 99114; M. Quaghebeur, Les deux «dogana», pp. 115-121; H. Bauchau, Les eaux vives, pp. 123-124; H. Bauchau, Pasiphaé, pp. 125-129; A. Marchetti, L'Euvre d'Henry Bauchau, pp. 131-136.

En 2002, Geneviève Henrot Sostero, de l'Université de Padoue, auteur d'un article sur «Mélopée Viking». Récit, prophétie, allégorie, paru dans le numéro monographique Henry Bauchau. Voix et vocation de l'écriture etc., cit., pp. 37-58 - un poème de la section Caste de guerriers du premier recueil de Bauchau (Géologie, Paris, Gallimard, 1958), réédité dans Poésie (1950-1986), Arles, Actes Sud, 1987 - essaie de mettre en relation, grâce aux pistes offertes par Bauchau même, le parcours analytique décelé par l'auteur à propos de son poème. Si les démarches analytiques croisées, de l'inconscient à l'écriture, du langage allégorique à l'approche philologique et linguistique du texte, ainsi qu'une approche s'appuyant sur la structure intratextuelle du poème, portent G. Henrot Sostero à admettre l'écart indéniable entre la lecture du critique, la philosophie de l'auteur et la signification possible dont le poème est d'une certaine manière investi au moment de l'élaboration, au point que le système de sens en est inévitablement changé, les recherches suivantes l'amènent à poursuivre son discours sur la poésie de Bauchau. En 2003, G. Henrot Sostero fait sortir le volume Henry Bauchau poète. Le Vertige du seuil (Genève, Droz), qui est encore aujourd'hui la seule monographie sur la poésie de l'écrivain. Même dans cette étude, ainsi que G. Henrot Sostero le souligne «c'est la quête d'une avancée et de ses étapes clés qui m'a poussé à reprendre les poésies dans le fil chronologique de leur première parution, tel qu'il fut reproduit dans Poésie (1950-1984)» ${ }^{48}$, la lecture textuelle suit, d'une part les itinéraires chronologiques des poèmes concernés à travers une première approche descriptive, de l'autre une démarche visant «à un éclairage latéral de discours ou de savoirs externes (psychanalyse, auto-biographie, auto-commentaires), qui ouvrent la porte à des 'interprétations' critiques plus ou moins légitimes, autorisées, vraisemblables, convaincantes... mais toujours, quelque part, indémontrables 'scientifiquement' parce que non falsifiables» ${ }^{49}$.

L'année suivante, avec E. Girardini, G. Henrot Sostero codirige Le corps à fleur de mots (Padova, Unipress, 2004), volume consacré à la thématique du corps dans les lettres françaises, de la Renaissance à l'époque contemporaine. Ici, une contribution de S. Bastesin, (Itinéraires génétiques du corps chez Henry Bauchau, pp. 113-133), vise à mettre en relief les rapports entre les images du corps et l'écriture sur le corps chez Bauchau. Mais, c'est encore en 2004, par la contribution à un Colloque tenu à Louvain-la-Neuve et à Dijon (18-20 octobre, 2004) ${ }^{50}$, que G. Henrot Sostero, Bauchaul Jouve: autour de l'allégorie, dans M. Watthee-Delmotte - J. Poirier (dir.), Pierre Jean

(47) Cf. ibid., p. 5 et pp. 6-7.

(48) En réalité, le travail de G. Henrot Sostero est axé sur un arc chronologique plus large par rapport à celui énoncé. En effet, ses analyses arrivent jusqu'à Exercice du matin (1999).

(49) Cf. G. Henrot Sostero, Henry Bauchau poète etc., cit., p. 13 et p. 12.

(50) Toujours en 2004 G. Henrot Sostero publie Henry Bauchau et la communion des eaux, dans L'eau: miroir des lettres belges?, «Nord'», 43 (avril 2004), pp. 109-121. En 2003 la chercheur fait sortir Henry Bauchau et la sémiose des astres: lecture de «Double zodiaque», dans AA. Vv., Les Constellations Impérieuses d'Henry Bauchau, cit., pp. 141-162. En 2002 Henry Bauchau et la Peauésie, «Agora», 2 (2002), pp. 115-134. En 1999 Le coureur de chagrins, dans Entretien avec Henry Bauchau, «L'Eil-de-Bœuf», 17 (avril 1999), pp. 27-32. En 1997 Henry Bauchau et le vertige du seuil, «Cahiers francophones d'Europe Centre-orientale», II, 
Jouve et Henry Bauchau: les voix de l'altérité, Dijon, Éditions Universitaires de Dijon («Écritures»), 2006, pp. 79-96, part des propositions théoriques de M. Bonhomme, axées sur une «récente révision de la rhétorique par la pragmatique cognitive» pour essayer «de relever, d'identifier et de classer les éléments qui, chez Jouve et chez Bauchau, peuvent nous induire à reconnaître - ou supposer - une allégorie et tenter la construction d'un sens second» ${ }^{51}$. En 2006, elle participe à un Colloque avec La Voix de l'ombre. Pouvoirs du rêve dans les journaux d'Henry Bauchau, dans Henry Bauchau et le (contre)-pouvoir de la parole, («Actes du Colloque International de Louvain-la-Neuve, 24-26 avril 2006»), intervention publiée dans «Nu(e)», 35 (mars 2007), pp. 249-260, tandis qu'en 2009 dans C. Mayaux - M. Watthee-Delmotte (dir.), Henry Bauchau, écrire etc., pp. 125-146, sort un de ses travaux Le Moi, le groupe et la peau plurielle. Henry Bauchau et «Le Régiment Noir», mais cette fois-ci il s'agit d'une étude autour de la prose de l'écrivain. En 2011, toujours dans Henry Bauchau, «Phœnix. Cahiers littéraires internationaux», 2 (avril 2011), pp. 29-34, elle fait sortir Les vertes tiges de l'écriture poétique. En tout cas, G. Henrot Sostero, poursuivant sa démarche scientifique sur la poésie bauchalienne, en 2012 publie, dans la «Revue Internationale Henry Bauchau. L'écriture à l'écoute», 4 (20112012), pp. 68-77, un article («Architectures de louange». Trame et chaîne d'une traduction) qui se base sur l'analyse du poème «Architectures etc.» paru dans Henry Bauchau, «Phœnix. Cahiers littéraires internationaux», 2 (avril 2011), pp. 22-23, un poème absent dans les recueils de Bauchau publiés jusqu'à présent. Après avoir reconstruit le contexte idéologique dans lequel s'insèrent les dynamismes d'élaboration poétique, liés à la dictée inconsciente de Bauchau, G. Henrot Sostero, à la lumière de considérations précises sur le système textuel (prosodique, allégorique et sémique) du poème en question, sur sa structure axiologique et sur les figures thématiques, voire allégoriques du texte, propose «un projet de traduction comme 'poétique expérimentale' (Meschonnic), qui appuie le balancement binaire entre les motifs (château/abbaye), les axiomatiques (violence/paix) et les rythmes (martelé/distendu) $\rangle^{52}$. Un projet de traduction qui offre de ce poème une édition avec commentaire.

En 2009, l'une des majeures spécialistes de l'œuvre de Bauchau, M. WattheeDelmotte (L'immaginario dell'Oriente in Bauchau: andare verso l'Altro per trovare Sé, dans Le frontiere dell'alterità, a cura di P. Proietti e R. Boccali, Palermo, Sellerio Editore, 2009, pp. 122-131), fait sortir cet article en Italie, le seul de toute son activité scientifique dans notre territoire.

Continuant cette rétrospective, nous rappelons que E. Surmonte, après avoir concentré son attention, en 2006, sur le personnage d'Antigone chez Bauchau, par un travail publié en 2008 (Per una 'nuova' Antigone, «mendiante définitive». Interpretazioni del XX-XXI secolo, dans AA. Vv., Il personaggio. Figure della dissolvenza e della permanenza, «Atti del Convegno dell'Associazione per gli Studi di Teoria e Storia Comparata della Letteratura», 14-16 settembre 2006, a cura di Ch. Lombardi, Alessandria, Edizioni dell'Orso, 2008, pp. 109-115), et, en 2007, sur le personnage d'CEdipe, par une étude portant en titre 'La barque d'Edipe' di Henry Bauchau, «Prosp'ero», XIV (2007), pp. 175-193, et aussi sur quelques Antigones du xx siècle, parmi lesquelles l'Antigone de Bauchau (Dall'archetipo al personaggio-persona. Di alcune riscritture e letture contemporanee dell' Antigone", «Annali. Sezione Romanza», XLIX, 1 (2007), pp. 47-83), publie, en 2011, Antigone, la Sphinx d'Henry Bauchau. Les enjeux d'une création, Bruxelles, Peter Lang («Documents pour l'Histoire des

7-8 (1997), pp. 139-161.

(51) Cf. ID., Bauchau/Jouve etc., cit., p. 79 et p. 81.

(52) Cf. ID., Architectures de louange ect., cit., 
Francophonies / Europe», n²4), avec Préface de M. Quaghebeur. Il s'agit de la première monographie sur le roman éponyme, dont l'intérêt porte surtout sur le matériel inédit consulté et analysé. En particulier, Surmonte étudie la première version de l'Antigone de Bauchau (cahiers 1, 2, 3), contenue dans les Archives et Musée de la Littérature de Bruxelles. Elle relève des variantes importantes, par rapport à la version consignée par Bauchau à la Maison d'Édition (Actes Sud) pour la publication de 1997. Ainsi que M. Quaghebeur le souligne, en préfacier de cette monographie, «la démarche exégétique de ce livre s'est dirigée vers la première mouture du roman précieusement déposée par l'auteur aux Archives et Musée de la Littérature. Elle s'est d'autre part portée vers le corpus bauchalien qui précède Antigone. En ce compris, et avec une acuité toute particulière, à travers la lecture attentive de l'admirable Régiment Noir, roman capital de l'œuvre» ${ }^{53}$. La bibliographie très riche (pp. 337-417) reste, même si en l'espace de deux ans ont paru de nombreux travaux, un utile instrument de recherche.

Assez récemment, en suivant la filière des réécritures du mythe d'Antigone, nous nous sommes occupé de la poétique de Bauchau. Dans une perspective analytique concernant des enquêtes structurelles portant sur un écrit inédit de l'auteur, nous avons essayé d'argumenter un principe théorique de traduction, formulé par Bauchau (M. MastroIANNI, Un écrit inédit d'Henry Bauchau: la 'traduction' d'une idée préliminaire pour Edipe sur la route, «Revue Internationale Henry Bauchau. L'écriture à l'écoute», 4 (2011-2012), pp. 130-146). Partant aussi d'un travail axé sur certains des dynamismes poétiques de son écriture, toujours envisagés à travers quelques corollaires argumentés par l'auteur, et focalisant notre attention sur un substrat lexical où nous avons relevé quelques tournures appartenant au discours théologique (Simmetrie e intertestualità teoretiche. Henry Bauchau fra dinamiche di elaborazione poetica e stilemi teologici, «Studi Francesi», 155 (maggio-agosto 2008), pp. 336-359), nous avons essayé de déceler - encore une fois dans une perspective d'élaboration poétique - les rapports, directs ou indirects, entre certaines réflexions de Höderlin et quelques axes herméneutiques de Bauchau, renvoyant à la notion heideggérienne de Dasein (D'une poétique paradoxale autour d'un insaisissable Protée? Henry Bauchau, écrivain dans le monde, dans C. Mayaux - M. Watthee-Delmotte (dir.), Henry Bauchau, écrire pour habiter le monde, cit., pp. 325-346). Toujours dans le sillage de cet intérêt pour les réflexions théoriques de Bauchau, sous-jacentes à son écriture et aux principes d'élaboration poétique qui la maîtrisent, nous avons concentré nos recherches sur le 'théoricien' Bauchau (Henry Bauchau teorico: riflessioni metalettarie tra scrittura poetica, sogno e psicanalisi, dans «De l'Ordre et de l'Aventure». Langue, littérature, francophonie. Hommage à Giovanni Dotoli, par P. Brunel, Ph. Desan, A. Rey, J. Pruvost, Paris, Hermann, 2013, sous presse).

Encore au sujet de l'élaboration poétique de cet auteur, nous avons mené des enquêtes sur les rapports entre La Déchirure et La Grande Muraille. Journal de «La Déchirure» (1960-1965), Arles, Actes Sud, 2005 (Appunti su «La Déchirure» di Henry Bauchau: memoria dell'infanzia, figura materna e psicanalisi, dans La rappresentazione della madre nella letteratura francese del Novecento, a cura di D. Cecchetti e M. Mastroianni, «Studi Francesi», 165 (settembre-dicembre 2011), pp. 579-588). Des enquêtes qui se sont croisées avec des recherches sur la valeur allégorique et symbolique du personnage de la mère chez Bauchau, dont les résultats ont aboutit dans la publication d'une monographie («La Déchirure» di Henry Bauchau. Una rappresenta-

p. 74 .

(53) Cf. M. Quaghebeur, Préface, dans E. Sur-
MONTE, Antigone, la Sphinx etc., cit., pp. 19-28, ici p. 21. 
zione della madre: allegoria dell'incontro e spazio di elaborazione poetica, Alessandria, Edizioni dell'Orso, 2013).

En guise de conclusion, nous soumettons au chercheur, par ordre chronologique,

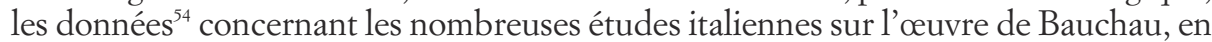
souhaitant que la grille ici proposée puisse fonctionner en tant qu'instrument bibliographique pour des recherches futures:

\begin{tabular}{|c|c|c|}
\hline $\begin{array}{l}\text { ANNÉE DE } \\
\text { PUBLICATION }\end{array}$ & AUTEUR & TITRE \\
\hline 1993 & A. Marchetti & $\begin{array}{l}\text { Edipe sur la route d'Henry } \\
\text { Bauchau. Le langage poétique } \\
\text { et sa provenance, dans C. VAN } \\
\text { RoGGER ANDREUCCI (dir.), Les } \\
\text { nowveaux courants poétiques } \\
\text { en France et en Grèce en } \\
\text { 1970-1990, Actes du Colloque } \\
\text { International, Thessalonique } \\
\text { (21-24 septembre 1993) }\end{array}$ \\
\hline
\end{tabular}

1993

1993

1995

1995

1997

1998
A. Marchetti La fedeltà dell'ascolto

Ch. Elefante

La responsabilité d'Édipe, du multiple à l'un, dans AA. Vv., Henry Bauchau. Un écrivain, une ceuvre, Atti del Centro di Studi sulla letteratura belga di lingua francese, Terzo Seminario Internazionale, Noci (8-10 novembre 1991)

Les rêves dans "CEdipe sur la route» d'Henry Bauchau, dans Rêve et poésie

A. MARChetti

Ch. Elefante

La scrittura del sogno della scrittura. A proposito di Henry Bauchau, dans A. SONCINI FratTA (dir.), Jeux de langue, jeux d'écriture

G. Henrot Sostero Henry Bauchau et le vertige du seuil

A. Marchetti
La traduction comme exode. Notes en marge de la traduction $d^{\prime}$ «Edipe sur la route»

\section{LIEU/MAISON \\ D'ÉDITION/REVUE}

Pau, Presses Universitaires de Pau, pp. 249-256

«In forma di parole», 4 (ottobre-dicembre 1993), pp. 149-151

Bologna, Editrice CLUEB, pp. 313-333

«Recherches et Travaux», 47 (1995), pp. 203-217

Bologna, Editrice CLUEB, pp. $129-151$

«Cahiers francophones d'Europe CentreOrientale», II, 7-8 (1997), pp. 139-161

«Cahiers Henry Bauchau», 2 (octobre 1998), pp. 36-45
(54) Puisque nous fournissons les données complètes des volumes cités, au fil de notre discours, par exemple celles qui concernent les collections ou, éventuellement, les noms des directeurs de ces mêmes collections ou les noms des chercheurs par les soins de qui tel ou tel volume a été édité, les études mentionnées dans la grille ici proposée ne renvoient plus à ces ultérieures références bibliographiques. 
1999

2000

2000

2002

2002

2002

2003

2003

2003

2003

2004

2004

2004
G. Henrot Sostero Le coureur de chagrins, dans Entretien avec Henry Bauchau

Ch. Elefante

De l'Edipe travesti à la renaissance du Père en Amérique, la patrie mythique des fils

Ch. Elefante

Tradurre «La Déchirure» di Henry Bauchau. Metodo e prassi

A. MARChetTI Henry Bauchau. Voix et vocation (a cura di)

de l'écriture

G. Henrot Sostero «Mélopée viking». Récit, prophétie, allégorie, dans Henry Bauchau. Voix et vocation de l'écriture

G. Henrot Sostero Henry Bauchau et la Peau-ésie

A. MARCHETTI

Le labyrinthe: mythe de l'écriture, écriture du mythe, dans AA. Vv., Les Constellations Impérieuses d'Henry Bauchau, Colloque de Cerisy-la-Salle (21-31 juillet 2001), Actes publiés sous la direction de M. Quaghebeur

Ch. Elefante

«Amour Diotime»: une lecture intertextuelle, dans AA. Vv., Les Constellations Impérieuses d'Henry Bauchau, cit.

G. Henrot Sostero Henry Bauchau et la sémiose des astres: lecture de «Double zodiaque», dans AA. Vv., Les Constellations Impérieuses d'Henry Bauchau, cit.

G. Henrot Sostero Henry Bauchau poète. Le Vertige du seuil

Ch. Elefante

Henry Bauchau e la scrittura, tra realtà, inconscio e vita onirica

Ch. Elefante

Henry Bauchau et Jean El Moubouv Amrouche: bistoire d'une amitié poétique, dans Aa. Vv., Henry Bauchau, une poétique de l'espérance, Actes $\mathrm{du}$ Colloque International, Metz (6-8 novembre 2002), sous la direction de P. Halen R. Michel - M. Michel

G. Henrot Sostero Henry Bauchau et la communion des eaux, dans L'eau: miroir des lettres belges?

S. BASTESIN
«L'CEil-de-Bœuf», 17 (avril 1999), pp. 27-32

«Cahiers Henry Bauchau», 3 (juin 2000), pp. 12-20

Torino, L'Harmattan Italia

«Francofonia», 42

(primavera 2002), pp. 136

«Francofonia», 42 (primavera 2002), pp. 37-58

«Agora», 2 (2002), pp. 115 134

Bruxelles, Archives et Musées de la Littérature, 2003, pp. 288-306

pp. $212-237$

pp. $115-134$

Genève, Droz

«Tratti», 67 (autunno 2004), pp. 6-17

Berne, Peter Lang, pp. 41 62

«Nord'», 43 (avril 2004), pp. $109-121$

Padova, Unipress, pp. 113133 
Giardini - G. HenRot Sostero, (dir.), Le corps à fleur de mots

2006

G. Henrot Sostero Bauchau/Jouve: autour de l'allégorie, dans M. WatTHEEDelmotte - J. Poirier (dir.), Pierre Jean-Jouve et Henry Bauchau: les voix de l'altérité

2006 G. Henrot Sostero La Voix de l'ombre. Pouvoirs du rêve dans les journaux d'Henry Bauchau, dans Henry Bauchau et le (contre)-pouvoir de la parole

M. MASTROIANNI

M. MASTROIANNI
M. WatTheEDeLmotte 'La barque d'Edipe' di Henry
Bauchau

Per una 'nuova' Antigone, «mendiante définitive». Interdans Aa.Vv., Il personaggio. Figure della dissolvenza e della permanenza, Atti del Convegno dell'Associazione per gli Studi di Teoria e Storia Comparata della Letteratura, Torino (14-16 settembre 2006)

Simmetrie e intertestualità teoretiche. Henry Bauchau fra dinamiche di elaborazione poetica e stilemi teologici

D’une poétique paradoxale autour d'un insaisissable Protée? Henry Bauchau, écrivain dans le monde, dans C. MAYAUX M. Watthee-Delmotte (dir.), Henry Bauchau, écrire pour babiter le monde

Ch. Elefante La voix du corps, la voix féminine dans l'aeuvre narrative d'Henry Bauchau, dans C. Mayaux - M. Watthee-Delмотте (dir.), Henry Bauchau, écrire pour habiter le monde

L'immaginario dell'Oriente in Bauchau: andare verso l'Altro per trovare Sé, dans P. ProietTI - R. BOCCALI (a cura di), Le frontiere dell'alterità

Le Moi, le groupe et la peau plurielle. Henry Bauchau et «Le Régiment Noir», dans C. MaYAuX Henry Baucbau, écrire pour babiter le monde

E. Surmonte Antigone, la Sphinx d'Henry Bauchau. Les enjeux d'une création pretazioni del XX-XXI secolo, - M. WattheE-Delmotte (dir.),
Dijon, Éditions Universitaires de Dijon, pp. 79-96

«Nu(e)», 35 (mars 2007), pp. $249-260$

«Prosp'ero», XIV (2007), pp. $175-193$

Alessandria, Edizioni dell'Orso, pp. 109-115

«Studi Francesi», 155 (maggio-agosto 2008), pp. 336359

Saint-Denis, Presses Universitaires de Vincennes, pp. 325-346

Vincennes, Presses Universitaires de Vincennes, pp. 147-160

Palermo, Sellerio Editore, pp. 122-131

Vincennes, Presses Universitaires de Vincennes, pp. 125-146

Bruxelles, Peter Lang 
2011

G. HenRot SOSTERO

Les vertes tiges de l'écriture poétique, dans Henry Bauchau

2011

M. MASTROIANNI

2012

2012

2013

2013
M. MASTROIANNI

G. Henrot Sostero

M. Mastroianni

M. MASTROIANNI
Appunti su «La Déchirure» di Henry Bauchau: memoria dell'infanzia, figura materna $e$ psicanalisi, dans La rappresentazione della madre nella letteratura francese del Novecento

Un écrit inédit d'Henry Bauchau: la 'traduction' d'une idée préliminaire pour Edipe sur la route

«Architectures de louange». Trame et chaine d'une traduction

Henry Bauchau teorico: riflessioni metaletterarie tra scrittura poetica, sogno e psicanalisi, dans P. BRunel - Ph. Desan - A. Rey - J. Pruvost (dir.), «De l'Ordre et de l'Aventure». Langue, littérature, francophonie. Hommage à Giovanni Dotoli

«La Déchirure» di Henry Bauchau. Una rappresentazione della madre: allegoria dell'incontro e spazio di elaborazione poetica
«Phoenix. Cahiers littéraires internationaux», 2 (avril 2011), pp. 29-34

«Studi Francesi», 165 (settembre-dicembre 2011), pp. $579-588$

«Revue Internationale Henry Bauchau. L'écriture à l'écoute», 4 (2011-2012), pp. 130-146.

«Revue Internationale Henry Bauchau. L'écriture à l'écoute», 4 (2011-2012), pp. 68-77

Paris, Hermann, 201355

Alessandria, dell'Orso, 2013
Edizioni

En guise de conclusion de notre discours, nous pouvons relever que, en l'espace de vingt ans (de 1993 à 2013), les études critiques en Italie, concernant l'œuvre d'Henry Bauchau, se sont organisé de manière cohérente. Ce qui est vrai au sujet de la matière mythique, ainsi que des problématiques gravitant autour de son élaboration. Par contre, ce sont encore très rares les recherches qui portent sur les recueils poétiques de l'auteur. Il s'agit, en effet, d'une donnée remarquable à la fois au niveau des études nationales et au niveau des études internationales. De même, s'il reste beaucoup de travail à faire - surtout sur les documents manuscrits inédits, conservés aux Archives et Musée de la Littérature de Bruxelles et au Fonds Henry Bauchau de Louvain-la-Neuve -, il faut souligner qu'il manque encore, en Italie comme à l'étranger, des enquêtes systématiques portant sur les journaux de Bauchau, sur les rapports tissés de la part de l'auteur d'une œuvre à l'autre, mais, surtout sur ses écrits théoriques et sur les liens d'intertextualité existant entre ses différents journaux intimes. Il s'agirait, dans ce cas particulier, de relations nodales importantes, dans la perspective de la recherche thématique, tout comme dans celle des références théoriques et autobiographiques.

(55) Puisque cet article est, en ce moment, sous presse, il nous a été impossible de connaître le numéro des pages qui le concernent. 
Les études sur ces rapports intertextuels seraient encore plus intéressantes si elles étaient menées à l'intérieur d'un cadre historiographique précis, construit sur un axe temporel chronologiquement consécutif, de façon qu'il puisse informer sur les évolutions effectives concernant le tissu idéologique et thématique de l'auteur, faisant constamment appel à un imaginaire en mouvement. En particulier, nous semble-t-il, les recherches sur la production diaristique de Bauchau pourraient aussi nous informer, pour la première fois, sur le rôle exercé par ses sources, directes et indirectes, dénoncées ou cachées, et sur un genre d'écriture, autour de laquelle cet écrivain a construit un laboratoire poétique tout à fait original. 